\title{
Slowing of paroxysmal tachycardia with loss of functional bundle-branch block
}

\author{
ROBERT A BAUERNFEIND, BORIS STRASBERG, KENNETH M ROSEN† \\ From the Section of Cardiology, Department of Medicine, Abraham Lincoln School of Medicine, University of Illinois \\ College of Medicine, Chicago, Illinois, USA
}

SUMMARY Electrophysiological studies in a patient with paroxysmal supraventricular tachycardia disclosed anterograde dual atrioventricular nodal pathways, and a concealed left-sided anomalous atrioventricular pathway which was used as the retrograde limb of two circus movement tachycardias. Tachycardia No.1, reflecting anterograde fast pathway conduction, was characterised by functional left bundle-branch block and a stable cycle length of 330 ms. Paroxysmal loss of bundlebranch block resulted in tachycardia No.2, which reflected anterograde slow pathway conduction, and was characterised by narrow QRS and a stable cycle length of $355 \mathrm{~ms}$. Tachycardia No.2 had a longer cycle length than tachycardia No.l because the increment in AH interval (slow pathway instead of fast pathway conduction) more than compensated for the decrement in ventriculoatrial interval (narrow QRS instead of bundle-branch block).

The mechanism of paroxysmal supraventricular tachycardia can usually be elucidated by detailed cardiac electrophysiological studies. ${ }^{1}$ An important diagnostic clue is frequently provided by paroxysmal loss of functional bundle-branch block during induced supraventricular tachycardia. When the loss of bundle-branch block results in a decrement in ventriculoatrial interval, a diagnosis of atrioventricular re-entry using an anomalous atrioventricular pathway ipsilateral to the blocked bundle-branch is strongly suggested. ${ }^{2}$ The decrement in ventriculoatrial interval is usually reflected by a decrement in the cycle length of tachycardia. ${ }^{3}$ The decrement in cycle length, however, may be relatively small, because an increment in AH interval may partially compensate for the decrement in ventriculoatrial interval. ${ }^{2}$

We report a patient in whom paroxysmal loss of functional bundle-branch block during induced supraventricular tachycardia resulted in an increment in cycle length of tachycardia. The mechanism of this unusual observation is discussed.

\section{Case report}

The patient was a 33-year-old woman with mitral

Supported in part by a NHLBI Institutional Training grant, and research grants. tDr Rosen died after the paper was accepted. valve prolapse and recurrent paroxysmal supraventricular tachycardia. After informed written consent was obtained, an electrophysiological study was performed, using standard techniques and definitions. 1 Recordings during sinus rhythm (rate 67 beats/min) disclosed a short AH interval $(41 \mathrm{~ms}$, normal 54 to 130) and normal PA and HV intervals (36 and $52 \mathrm{~ms}$, respectively). Atrial pacing from multiple sites failed to show evidence of ventricular pre-excitation.

Extrastimulus testing from the high right atrium (driven cycle length $500 \mathrm{~ms}$ ) showed discontinuous $A_{1}-A_{2}, A_{2}-H_{2}$ and $A_{1}-A_{2}, H_{1}-H_{2}$ conduction curves, suggesting the presence of anterograde dual (fast and slow) atrioventricular nodal pathways. ${ }^{4}$ The fast pathway was characterised by $\mathrm{A}_{2}-\mathrm{H}_{2}$ intervals of 60 to $100 \mathrm{~ms}$ and an effective refractory period of $380 \mathrm{~ms}$, whereas the slow pathway was characterised by $\mathrm{A}_{2}-\mathrm{H}_{2}$ intervals of 165 to $260 \mathrm{~ms}$ and an effective refractory period of $270 \mathrm{~ms}$. Extrastimuli which conducted over the slow pathway with an $\mathrm{A}_{2}-\mathrm{H}_{2}$ interval of $210 \mathrm{~ms}$ or longer resulted in induction of tachycardia No.2 (see below).

Incremental pacing from the high atrium at rates of 100 to 130 beats/min disclosed AH intervals of 60 to $90 \mathrm{~ms}$. At an atrial paced rate of 140 beats $/ \mathrm{min}$, two sets of stable AH intervals (100 to $220 \mathrm{~ms}$ ) were observed, again suggesting the presence of antero- 
grade dual atrioventricular nodal pathways. At atrial paced rates of 150 to 170 beats/min, AH intervals were 220 to $270 \mathrm{~ms}$. Termination of atrial pacing during conduction over the slow pathway resulted in induction of tachycardia No.2 (see below).

Ventricular incremental pacing, at rates of 100 to 240 beats/min, showed intact ventriculoatrial conduction with a fixed ventriculoatrial conduction time and an eccentric sequence of retrograde atrial activation (left atrium depolarised first). These findings suggested the presence of a concealed left-sided anomalous atrioventricular pathway. ${ }^{1}$ Termination of ventricular pacing at a rate of 240 beats $/ \mathrm{min}$ resulted in induction of tachycardia No.1 (see below).

\section{INDUCED SUPRAVENTRICULAR TACHYCARDIAS}

Two distinct tachycardias were induced by programmed stimulation. Tachycardia No.2 (induced many times by atrial stimulation) was always characterised by narrow QRS complexes and a stable cycle length of $355 \mathrm{~ms}$. During this tachycardia, the sequence of atrial activation was eccentric (distal coronary sinus, proximal coronary sinus, high right atrial, and low septal right atrial electrograms followed the onset of QRS complexes by $85,105,150$, and $175 \mathrm{~ms}$, respectively), and the atria could be reset by ventricular extrastimuli introduced when the His bundle was being depolarised anterogradely, suggesting that this tachycardia reflected a circus movement using the anomalous pathway as the retrograde limb. ${ }^{1}$ The AH interval was relatively long $(130 \mathrm{~ms})$, suggesting that the anterograde limb of the circus movement was the slow atrioventricular nodal pathway (see Discussion).

Tachycardia No.1 (induced only once, by ventricular pacing) was characterised by functional left bundle-branch block and a stable cycle length of 330 ms. The sequence of atrial activation during this tachycardia was identical to the sequence observed during tachycardia No.2, suggesting that tachycardia No.1 also reflected a circus movement which used the anomalous pathway as the retrograde limb. During this tachycardia, the AH interval was very short (30 $\mathrm{ms}$ ), suggesting that the anterograde limb of the circus movement was the fast atrioventricular nodal pathway (see Discussion).

During tachycardia No.1 paroxysmal loss of functional left bundle-branch block resulted in tachycardia No.2 (Fig.). This loss of bundle-branch block caused shortening of the intervals from onset of QRS complexes to each of the atrial electrograms by $75 \mathrm{~ms}$ (from 160 to $85 \mathrm{~ms}$ for the distal coronary sinus electrogram). The cycle length of tachycardia, however, did not shorten but lengthened by $25 \mathrm{~ms}$. The lengthening of the tachycardia cycle length reflected an increment in AH interval of $100 \mathrm{~ms}$ (from 30 to 130 ms) which more than compensated for the decrement

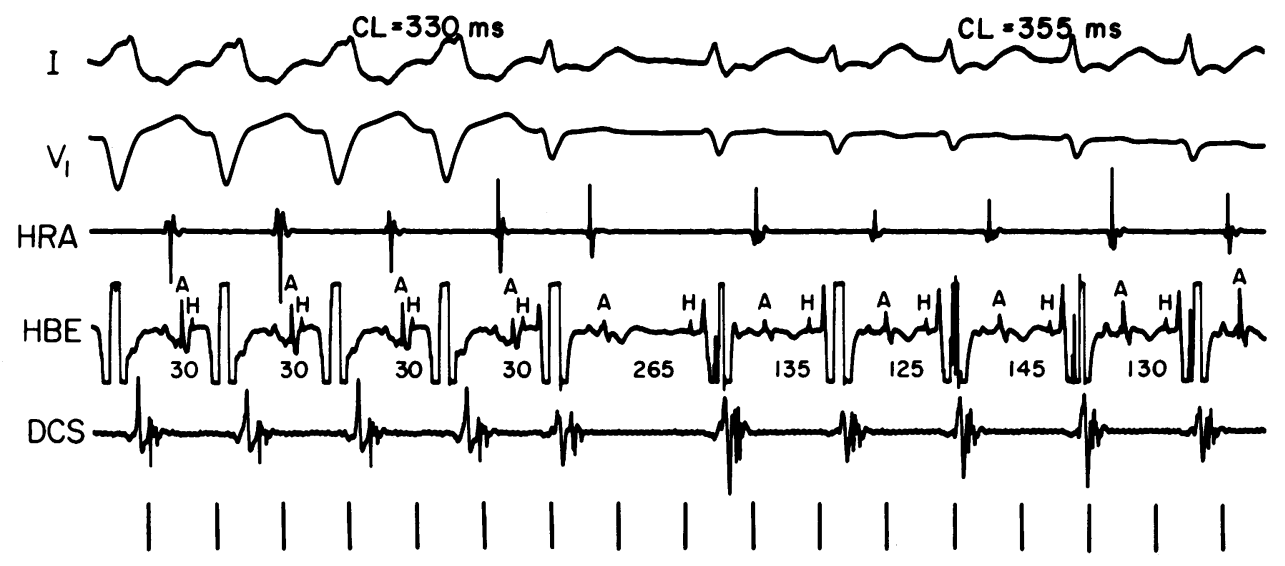

Fig. Paroxysmal loss of functional left bundle-branch block during atrioventricular re-entrant tachycardia. Electrocardiographic leads I and VI, as well as high right atrial (HRA), His bundle (HBE), and distal coronary sinus (DCS) electrograms, are shown. AH intervals are shown below HBE. Time lines (bottom) are at $200 \mathrm{~ms}$ interoals. The furst four beats of re-entrant tachycardia are conducted with lefi bundle-branch block and have cycle length (CL) $330 \mathrm{~ms}$. Note that the sequence of retrograde atrial activation is eccentric, proceeding from DCS to $H R A$ and then to the low septal right atrium $(H B E)$, and that the $A H$ interval is 30 ms (fast atrioventricular nodal pathway conduction). At the fifth beat there is sudden loss of left bundle-branch block, resulting in shortening of the ventriculoatrial interoal. The tachycardia continues, with the last five beats of paroxysmal supraventricular. tachycardia being conducted normally and having CL $355 \mathrm{~ms}$. Note that the sequence of retrograde atrial activation is unchanged, but that the AH interoal is $130 \mathrm{~ms}$ (slow atrioventricular nodal pathway conduction). 
in ventriculoatrial interval. The $\mathrm{HV}$ interval was 50 ms during each of the tachycardias.

\section{Discussion}

Occasional patients have dual atrioventricular pathways coexisting with anomalous atrioventricular pathways. ${ }^{56}$ These patients have the potential for several distinct circus movement tachycardias. ${ }^{56}$ Our patient had anterograde dual atrioventricular nodal pathways, and a concealed left-sided anomalous atrioventricular pathway which was used as the retrograde limb of two circus movements. One circus movement used the slow atrioventricular nodal pathway (AH intervals of $130 \mathrm{~ms}$ ) as the anterograde limb, and was conducted with normal QRS complexes. The other circus movement used the fast atrioventricular nodal pathway ( $\mathrm{AH}$ intervals of $30 \mathrm{~ms}$ ) as the anterograde limb, and was conducted with left bundlebranch block. Fast and slow pathway AH intervals were shorter during these circus movements than during high right atrial pacing. This probably reflected the fact that during these circus movements input to the atrioventricular node was from the left atrium, as AH intervals are significantly shorter during coronary sinus pacing than during high right pacing at equivalent rates. ${ }^{7}$

Our patient presented an almost unique finding. During induced atrioventricular re-entrant tachycardia, paroxysmal loss of functional bundle-branch block resulted in sudden lengthening of the cycle length of tachycardia. The explanation for this finding is as follows. When the functional bundle-branch block was lost, the ventriculoatrial interval shortened, resulting in early atrial depolarisation and early anterograde input into the atrioventricular node. This early input found the fast atrioventricular nodal pathway refractory, and conducted anterogradely over the slow atrioventricular nodal pathway. Subsequent beats of the narrow QRS tachycardia also conducted over the slow pathway, probably because of retrograde concealment into the fast pathway. The cycle length of tachycardia increased because the increment in AH interval (slow pathway instead of fast pathway conduction) more than compensated for the decrement in ventriculoatrial interval (narrow QRS instead of left bundle-branch block).

We are aware of only one patient similar to ours. Akhtar and co-workers ${ }^{8}$ reported a patient with induced atrioventricular re-entrant tachycardia, during which loss of functional right bundle-branch block resulted in a $20 \mathrm{~ms}$ increase in cycle length of tachycardia, because the AH interval increased by 65 ms whereas the ventriculoatrial interval decreased by only $45 \mathrm{~ms}$. An explanation for this observation was not given. We suspect that their patient had anterograde dual atrioventricular nodal pathways, which were not shown during atrial extrastimulus testing because the fast pathway effective refractory period was shorter than the atrial functional refractory period at the cycle length employed.

Our observations provide an additional surface electrocardiographic clue to the diagnosis of a specific mechanism of paroxysmal supraventricular tachycardia. Slowing of tachycardia with loss of functional bundle-branch block (or speeding of tachycardia with the occurrence of functional bundle-branch block) suggests the possibility of anterograde dual atrioventricular nodal pathways coexisting with an anomalous atrioventricular pathway ipsilateral to the site of functional bundle-branch block.

\section{References}

1 Wu D, Denes P, Amat-y-Leon F, et al. Clinical, electrocardiographic and electrophysiologic observations in patients with supraventricular tachycardia. Am $\mathcal{f}$ Cardiol 1978; 41: 1045-51.

2 Pritchett ELC, Tonkin AM, Dugan FA, Wallace AG, Gallagher JJ. Ventriculoatrial conduction time during reciprocating tachycardia with intermittent bundlebranch block in Wolff-Parkinson-White syndrome. $\mathrm{Br}$ Heart 7 1976; 38: 1058-64.

3 Spurrell RAJ, Krikler DM, Sowton E. Retrograde invasion of the bundle branches producing aberration of the QRS complex during supraventricular tachycardia studied by programmed electrical stimulation. Circulation 1974; 50: 487-95.

4 Denes P, Wu D, Dhingra RC, Chuquimia R, Rosen KM. Demonstration of dual A-V nodal pathways in patients with paroxysmal supraventricular tachycardia. Circulation 1973; 48: 549-55.

5 Spurrell RAJ, Krikler D, Sowton E. Two or more intra $A-V$ nodal pathways in association with either a James or Kent extranodal bypass in 3 patients with paroxysmal supraventricular tachycardia. Br Heart f 1973; 35: 11322.

6 Amat-y-Leon F, Wyndham C, Wu D, Denes P, Dhingra RC, Rosen KM. Participation of fast and slow A-V nodal pathways in tachycardia complicating the WolffParkinson-White syndrome. Circulation 1977; 55: 663-8.

7 Amat-y-Leon F, Denes P, Wu D, Pietras RJ, Rosen KM. Effects of atrial pacing site on atrial and atrioventricular nodal function. Br Heart $\mathcal{J}$ 1975; 37: 576-82.

8 Akhtar M, Damato AN, Ruskin JN, et. al. Antegrade and retrograde conduction characteristics in three patterns of paroxysmal atrioventricular junctional reentrant tachycardia. Am Heart f 1978; 95: 22-42.

Requests for reprints to Dr Robert A Bauernfeind, Cardiology Section, University of Illonois Hospital, PO Box 6998, Chicago, Illinois 60680, USA. 